\title{
Wigner distributions of quarks for different polarizations
}

Received: date / Accepted: date

\begin{abstract}
We calculate quark Wigner distributions using the light-front wave functions in a dressed quark model. In this model, a proton target is replaced by a simplified spin- $1 / 2$ state, namely a quark dressed with a gluon. We calculate the Wigner distributions for different polarization configuration of quark and the target state in this model.
\end{abstract}

Keywords Wigner distributions · dressed quark model

\section{Introduction}

Generalized parton distributions (GPDs) and transverse momentum dependent parton distributions (TMDs) [1] give the three-dimensional picture of the hadronic structure. GPDs [2; 3] essentially can provide an understanding of the orbital angular momentum of partons inside the hadrons. GPDs and TMDs are encompassed in a correlation function which describes off-forward scattering amplitudes that were introduced in Ref. [4. For certain kinematics, if one calculates the matrix element at $z^{+}=0$ or $z^{-}=0$, one obtains a reduced matrix element called as generalized transverse momentum dependent parton distributions (GTMDs) 1; 4; 5. Wigner distributions are obtained by taking Fourier transform of GTMDs which are also called as mother distribution functions. The main goal is to understand the partonic structure of nucleon that can be achieved by means of position-momentum distributions like Wigner distributions. In a particular limit, these distributions can be related to the spin and angular momentum of quarks and gluons. Wigner distribution is defined as matrix elements between different nucleon states as a function of three position and three momentum of a quark or a gluon inside the nucleon in a particular polarization state.

Quark and gluon Wigner distribution as a function of three position and three momentum variables was first studied by authors in Refs. [6, 7] in which relativistic effects were neglected. Wigner distributions 8 do not give probabilistic interpretation as they are not positive definite. Only in the classical limit, they become positive definite. Inspite of this, a phase-space distribution are worth exploring as they can be related to the measurable quantities by integration over transverse position or momentum of the parton. The model dependent calculation of Wigner distributions may provide us information

Presented by Jai More at Light Cone 2016, 5-8 September 2016, IST, Universidade de Lisboa, Portugal.

Jai More · Asmita Mukherjee

Indian Institute of Technology, Bombay, Mumbai-400076, India.

E-mail: jai.more@phy.iitb.ac.in

Asmita Mukherjee

E-mail: asmita@phy.iitb.ac.in

Sreeraj Nair

Indian Institute of Science Education and Research, Bhopal, MP-462030, India.

E-mail: sreeraj_nair@iiserb.ac.in 
about the correlators of quarks and gluons in a nucleon. Especially, quark orbital angular momentum can be accessed from the Wigner distribution for unpolarized quarks in the longitudinally polarized nucleon $\left(F_{14}\right)$, ; 10, 11; 12, 13, 14. These calculations are also a boon in estimating relations of TMDs and GPDs using GTMDs in a certain limit. Wigner distributions have been studied in various phenomenological models [10, 14; 15, 16, 17, 18; 19, 20].

In this work, we investigate the quark Wigner distributions in light-front Hamiltonian formalism [21. In this formalism, one gets an intuitive picture of deep inelastic scattering for non-collinear, interacting and massive partons. The target state is a dressed quark state and is expanded in terms of light-front wavefunctions (LFWFs) which are the multiparton wavefunctions. So, in dressed quark model we replace the proton state as a quark dressed with a gluon which incorporates gluonic degrees of freedom in this perturbative model.

The plan of this paper is as follows. In Sec. 2 we calculate the Wigner distribution of quark. For different polarization configuration of quark and target state, one can define 16 Wigner distributions at the leading twist. All these Wigner distributions are calculated in light cone dressed quark model using Hamiltonian perturbation theory. In our model, we obtained 8 independent distributions and we illustrate here only 4 of them, a complete discussion of all the leading twist Wigner distributions can be found in 23. In Sec. 3 we give numerical results and three-dimensional plots of the independent distributions in momentum space, impact parameter space and mixed space. Finally, we summarize our results in Sec. 4

\section{Quark Wigner Distributions in Dressed Quark Model}

The Wigner distribution of quarks is defined as [1, 9]

$$
\rho^{[\Gamma]}\left(b_{\perp}, k_{\perp}, x, s, s^{\prime}\right)=\int \frac{d^{2} \Delta_{\perp}}{(2 \pi)^{2}} e^{-i \Delta_{\perp} . b_{\perp}} W^{[\Gamma]}\left(\Delta_{\perp}, k_{\perp}, x, s, s^{\prime}\right)
$$

where, $\boldsymbol{b}_{\perp}$ is the impact parameter space conjugate to momentum transfer $\left(\Delta_{\perp}\right)$ of a dressed quark in the transverse direction. GTMDs are defined through quark-quark correlator $W^{[\Gamma]}$ at a fixed light-front time as

$$
W^{[\Gamma]}\left(\Delta_{\perp}, k_{\perp}, x, s, s^{\prime}\right)=\left.\int \frac{d z^{-} d^{2} z_{\perp}}{2(2 \pi)^{3}} e^{i k . z}\left\langle p^{+}, \frac{\Delta_{\perp}}{2}, s\left|\bar{\psi}\left(-\frac{z}{2}\right) \Omega \Gamma \psi\left(\frac{z}{2}\right)\right| p^{+},-\frac{\Delta_{\perp}}{2}, s^{\prime}\right\rangle\right|_{z^{+}=0}
$$

The initial and final dressed quark states are defined in the symmetric frame, with the longitudinal momentum as $p^{+}$, the transverse momentum transfer is $\Delta_{\perp}$ and $s, s^{\prime}$ are the helicities of initial and final target state. The average four momentum of the quark is $k$, with $k^{+}=x p^{+}$, here $x$ is the longitudinal momentum fraction of the parton. $\Omega$ is the gauge link which is required for color gauge invariance and is chosen to be unity.

The state of a dressed quark with momentum $p$ and fixed helicity $s$ can be written in terms of multiparton wavefunctions (LFWFs) as the expansion of the Fock state [21]

$$
\begin{aligned}
\left|p^{+}, p_{\perp}, s\right\rangle= & \Phi^{s}(p) b_{s}^{\dagger}(p)|0\rangle+\sum_{s_{1} s_{2}} \int \frac{d p_{1}^{+} d^{2} p_{1}^{\perp}}{\sqrt{16 \pi^{3} p_{1}^{+}}} \int \frac{d p_{2}^{+} d^{2} p_{2}^{\perp}}{\sqrt{16 \pi^{3} p_{2}^{+}}} \sqrt{16 \pi^{3} p^{+}} \delta^{3}\left(p-p_{1}-p_{2}\right) \\
& \times \Phi_{s_{1} s_{2}}^{s}\left(p ; p_{1}, p_{2}\right) b_{s_{1}}^{\dagger}\left(p_{1}\right) a_{s_{2}}^{\dagger}\left(p_{2}\right)|0\rangle
\end{aligned}
$$

$\Phi^{s}(p)$ is the single quark state and $\Phi_{s_{1} s_{2}}^{s}\left(p ; p_{1}, p_{2}\right)$ is the quark-gluon LFWF. $s_{1}$ and $s_{2}$ are helicities of quark and gluon respectively. $\Phi^{s}(p)$ gives the wavefunction normalization of the quark. $\Phi_{s_{1} s_{2}}^{s}\left(p ; p_{1}, p_{2}\right)$ gives the probability amplitude to find a bare quark (gluon) with momentum $p_{1}\left(p_{2}\right)$ and helicity $s_{1}\left(s_{2}\right)$ inside the dressed quark. Using the Jacobi momenta

$$
k_{i}^{+}=x_{i} P^{+} \text {and } \quad k_{i}^{\perp}=q_{i}^{\perp}+x_{i} P^{\perp} \quad \text { so that } \quad \sum_{i} x_{i}=1, \quad \sum_{i} q_{i \perp}=0
$$

the two particle LFWF can be written in terms of boost invariant LFWF as

$$
\sqrt{P^{+}} \Phi\left(p ; p_{1}, p_{2}\right)=\Psi\left(x_{i}, q_{i}^{\perp}\right)
$$


Using the two particle LFWF 21] and two component formalism 22] at leading twist, one obtains only four Dirac operators $\Gamma=\left\{\gamma^{+}, \gamma^{+} \gamma^{5}, i \sigma^{+1} \gamma^{5}, i \sigma^{+2} \gamma^{5}\right\}$ which corresponds to Wigner distributions for unpolarized, longitudinally polarized and transversely polarized dressed quark. So the quark-quark correlator using two particle LFWFs for different polarization at twist-2 are given by

$$
\begin{aligned}
W^{\left[\gamma^{+}\right]}\left(\Delta_{\perp}, k_{\perp}, x, s, s^{\prime}\right) & =\sum_{\lambda_{1}^{\prime}, \lambda_{1}, \lambda_{2}} \Psi_{\lambda_{1}^{\prime} \lambda_{2}}^{* s^{\prime}}\left(x, q^{\perp}\right) \chi_{\lambda_{1}^{\prime}}^{\dagger} \chi_{\lambda_{1}} \Psi_{\lambda_{1} \lambda_{2}}^{s}\left(x, q^{\perp}\right) \\
W^{\left[\gamma^{+} \gamma^{5}\right]}\left(\Delta_{\perp}, k_{\perp}, x, s, s^{\prime}\right) & =\sum_{\lambda_{1}^{\prime}, \lambda_{1}, \lambda_{2}} \Psi_{\lambda_{1}^{\prime} \lambda_{2}}^{* s^{\prime}}\left(x, q^{\perp}\right) \chi_{\lambda_{1}^{\prime}}^{\dagger} \sigma_{3} \chi_{\lambda_{1}} \Psi_{\lambda_{1} \lambda_{2}}^{s}\left(x, q^{\perp}\right) \\
W^{\left[i \sigma^{+j} \gamma^{5}\right]}\left(\Delta_{\perp}, k_{\perp}, x, s, s^{\prime}\right) & =\sum_{\lambda_{1}^{\prime}, \lambda_{1}, \lambda_{2}} \Psi_{\lambda_{1}^{\prime} \lambda_{2}}^{* s^{\prime}}\left(x, q^{\perp}\right) \chi_{\lambda_{1}^{\prime}}^{\dagger} \sigma_{j} \chi_{\lambda_{1}} \Psi_{\lambda_{1} \lambda_{2}}^{s}\left(x, q^{\perp}\right)
\end{aligned}
$$

with $\sigma_{i}$ are the three Pauli matrices. Eqs. (6), (7) and (8) gives unpolarized, longitudinally polarized and transversely polarized GTMDs in terms of LFWFs. For various combinations of unpolarized (U), longitudinally polarized (L) and transversely polarized (T) target and quark states, the quark-quark correlators can be parametrized into 16 Wigner distributions [18 at leading twist. We denote Wigner distributions by $\rho_{\lambda, \lambda^{\prime}}$, where $\lambda$ and $\lambda^{\prime}$ represents polarization of the target state and quark respectively. In this model, we have 10 independent Wigner distributions out of which $\rho_{T T}^{j}\left(\boldsymbol{b}_{\perp}, \boldsymbol{k}_{\perp}, x\right)$ is zero with $j=1,2$ and $\rho_{L U}=\rho_{U L}$. However, we discuss only four independent Wigner distributions but the details of all the 10 Wigner distributions can be looked in Ref [23] and in that we have classified Wigner distribution in terms of polarization of the target states.

The unpolarized Wigner distribution

$$
\rho_{U U}\left(\boldsymbol{b}_{\perp}, \boldsymbol{k}_{\perp}, x\right)=\frac{1}{2}\left[\rho^{\left[\gamma^{+}\right]}\left(\boldsymbol{b}_{\perp}, \boldsymbol{k}_{\perp}, x, \hat{\boldsymbol{e}}_{z}\right)+\rho^{\left[\gamma^{+}\right]}\left(\boldsymbol{b}_{\perp}, \boldsymbol{k}_{\perp}, x,-\hat{\boldsymbol{e}}_{z}\right)\right]
$$

The unpolarized-longitudinally polarized Wigner distribution

$$
\rho_{U L}\left(\boldsymbol{b}_{\perp}, \boldsymbol{k}_{\perp}, x\right)=\frac{1}{2}\left[\rho^{\left[\gamma^{+} \gamma^{5}\right]}\left(\boldsymbol{b}_{\perp}, \boldsymbol{k}_{\perp}, x, \hat{\boldsymbol{e}}_{z}\right)+\rho^{\left[\gamma^{+} \gamma^{5}\right]}\left(\boldsymbol{b}_{\perp}, \boldsymbol{k}_{\perp}, x,-\hat{\boldsymbol{e}}_{z}\right)\right]
$$

The longitudinal Wigner distribution

$$
\rho_{L L}\left(\boldsymbol{b}_{\perp}, \boldsymbol{k}_{\perp}, x\right)=\frac{1}{2}\left[\rho^{\left[\gamma^{+} \gamma^{5}\right]}\left(\boldsymbol{b}_{\perp}, \boldsymbol{k}_{\perp}, x, \hat{\boldsymbol{e}}_{z}\right)-\rho^{\left[\gamma^{+} \gamma^{5}\right]}\left(\boldsymbol{b}_{\perp}, \boldsymbol{k}_{\perp}, x,-\hat{\boldsymbol{e}}_{z}\right)\right]
$$

The transverse Wigner distribution

$$
\rho_{T T}\left(\boldsymbol{b}_{\perp}, \boldsymbol{k}_{\perp}, x\right)=\frac{1}{2} \delta_{i j}\left[\rho^{\left[i \sigma^{+j} \gamma^{5}\right]}\left(\boldsymbol{b}_{\perp}, \boldsymbol{k}_{\perp}, x, \hat{\boldsymbol{e}}_{i}\right)-\rho^{\left[i \sigma^{+j} \gamma^{5}\right]}\left(\boldsymbol{b}_{\perp}, \boldsymbol{k}_{\perp}, x,-\hat{\boldsymbol{e}}_{i}\right)\right]
$$

The pretzelous Wigner distribution

$$
\rho_{T T}^{\perp}\left(\boldsymbol{b}_{\perp}, \boldsymbol{k}_{\perp}, x\right)=\frac{1}{2} \epsilon_{i j}\left[\rho^{\left[i \sigma^{+j} \gamma^{5}\right]}\left(\boldsymbol{b}_{\perp}, \boldsymbol{k}_{\perp}, x, \hat{\boldsymbol{e}}_{i}\right)-\rho^{\left[i \sigma^{+j} \gamma^{5}\right]}\left(\boldsymbol{b}_{\perp}, \boldsymbol{k}_{\perp}, x,-\hat{\boldsymbol{e}}_{i}\right)\right]
$$

Thus, the analytical expressions for the four Wigner distributions that we study are given by

$$
\begin{aligned}
& \rho_{U U}\left(\boldsymbol{b}_{\perp}, \boldsymbol{k}_{\perp}, x\right)=N \int d^{2} \Delta_{\perp} \frac{\cos \left(\Delta_{\perp} b_{\perp}\right)}{D\left(q_{\perp}\right) D\left(q_{\perp}^{\prime}\right)}\left[\frac{\left(4 k_{\perp}^{2}-\Delta_{\perp}^{2}(1-x)^{2}\right)\left(1+x^{2}\right)}{x^{2}(1-x)^{3}}+\frac{4 m^{2}(1-x)}{x^{2}}\right] \\
& \rho_{U L}\left(\boldsymbol{b}_{\perp}, \boldsymbol{k}_{\perp}, x\right)=N \int d^{2} \Delta_{\perp} \frac{\sin \left(\Delta_{\perp} b_{\perp}\right)}{D\left(q_{\perp}\right) D\left(q_{\perp}^{\prime}\right)}\left[\frac{4\left(k_{x} \Delta_{y}-k_{y} \Delta_{x}\right)(1+x)}{x^{2}(1-x)}\right] \\
& \rho_{L L}\left(\boldsymbol{b}_{\perp}, \boldsymbol{k}_{\perp}, x\right)=N \int d^{2} \Delta_{\perp} \frac{\cos \left(\Delta_{\perp} b_{\perp}\right)}{D\left(q_{\perp}\right) D\left(q_{\perp}^{\prime}\right)}\left[\frac{\left(4 k_{\perp}^{2}-\Delta_{\perp}^{2}(1-x)^{2}\right)\left(1+x^{2}\right)}{x^{2}(1-x)^{3}}-\frac{4 m^{2}(1-x)}{x^{2}}\right] \\
& \rho_{T T}\left(\boldsymbol{b}_{\perp}, \boldsymbol{k}_{\perp}, x\right)=N \int d^{2} \Delta_{\perp} \frac{\cos \left(\Delta_{\perp} b_{\perp}\right)}{D\left(q_{\perp}\right) D\left(q_{\perp}^{\prime}\right)}\left[\frac{2\left(4 k_{\perp}^{2}-\Delta_{\perp}^{2}(1-x)^{2}\right)}{x(1-x)^{3}}\right]
\end{aligned}
$$


where,

$$
\begin{gathered}
N=\frac{g^{2} C_{F}}{2(2 \pi)^{2}}, \quad C_{F} \text { is the color factor } \\
D\left(q_{\perp}\right)=\left[m^{2}-\frac{m^{2}+\left(k_{\perp}+\frac{\Delta_{\perp}(1-x)}{2}\right)^{2}}{x}-\frac{\left(k_{\perp}+\frac{\Delta_{\perp}(1-x)}{2}\right)^{2}}{1-x}\right] \\
D\left(q_{\perp}^{\prime}\right)=\left[m^{2}-\frac{m^{2}+\left(k_{\perp}-\frac{\Delta_{\perp}(1-x)}{2}\right)^{2}}{x}-\frac{\left(k_{\perp}-\frac{\Delta_{\perp}(1-x)}{2}\right)^{2}}{1-x}\right]
\end{gathered}
$$

We observed that, $\rho_{T T}^{\perp}\left(\boldsymbol{b}_{\perp}, \boldsymbol{k}_{\perp}, x\right)=0$ in this model.

\section{Numericals and plots of probability densities in three dimension}

As mentioned in the previous section, we study 4 independent Wigner distributions given in Eqs. 14 - 17 which are five dimensional function of $b_{x}, b_{y}, k_{x}, k_{y}$ and $x$. We integrate over the momentum fraction $x$ which can take values only between $(0,1)$, in the dressed quark model. We observe the behavior in the transverse momentum and impact parameter space of these 4 distributions. One may also look at the mixed distributions by integrating out transverse momentum and transverse position along the perpendicular transverse directions. In the mixed distribution one expects distribution to be real as the remaining variables are not restricted by Heisenberg's uncertainty principle. In this section, we show the numerical results and the plots of the quark Wigner distributions in transverse momentum space with $b_{\perp}=0.4 \mathrm{GeV}^{-1}$, transverse position space with $k_{\perp}=0.4 \mathrm{GeV}$ and mixed space. The integration limit of $\Delta_{\perp}$ should ideally be from zero to infinity. However, in the numerical integration,
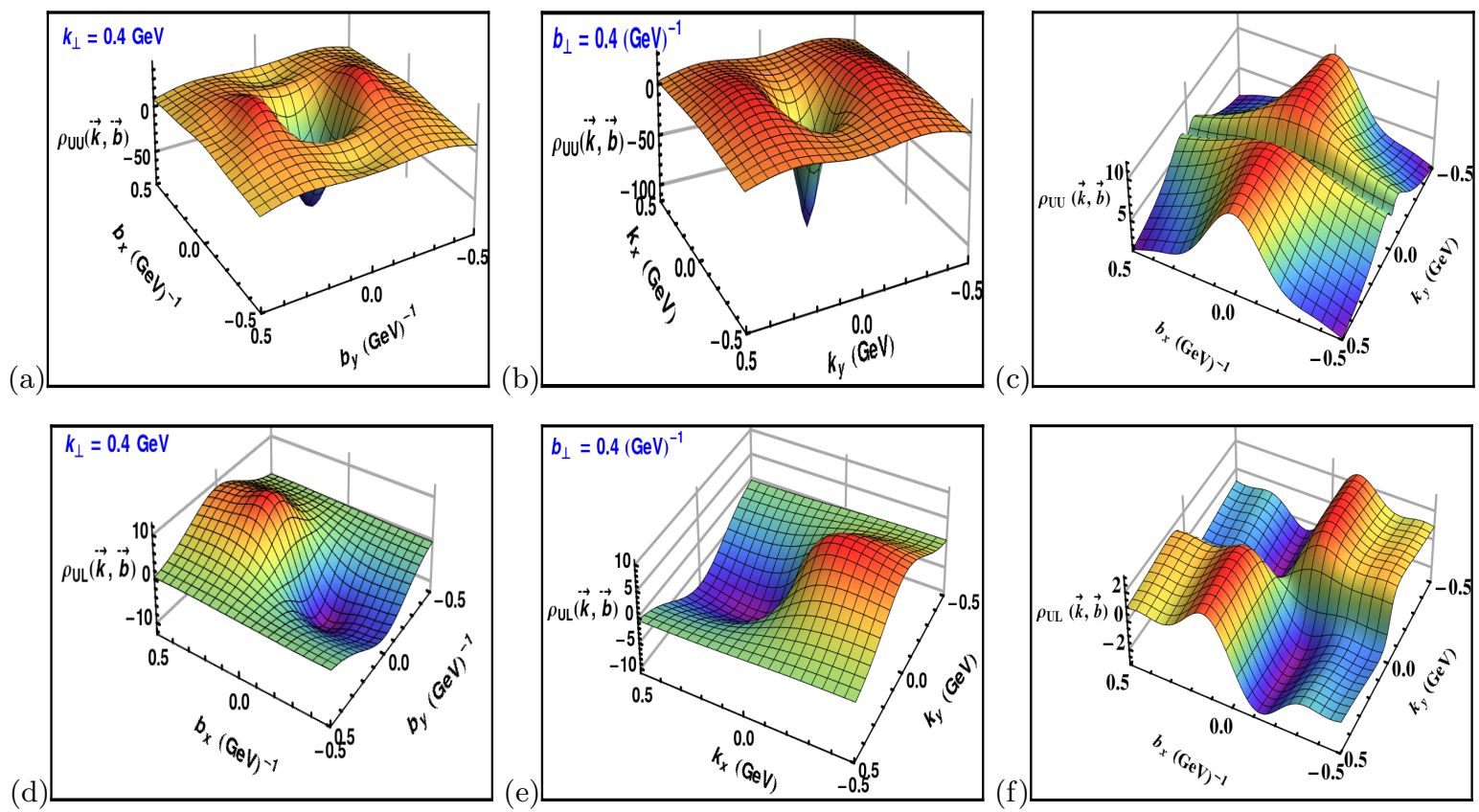

Fig. $13 \mathrm{D}$ plot of Wigner distributions $\rho_{U U}$ and $\rho_{U L}$ in $\boldsymbol{k}_{\perp}-$ space, $\boldsymbol{b}_{\perp}-$ space and $b_{x}-k_{y}$ space with $\Delta_{\perp \max }=$ $20 \mathrm{GeV}$

we have used a cutoff on $\Delta_{\perp}$ integration, $\Delta_{\perp \max }=20 \mathrm{GeV}$. It is to be noted that in the previous work [14; 15], a lower value of $\Delta_{\perp \max }$ was used and the results depend on this cutoff. We use Levin's method to perform numerical integration over $x$ and $\Delta_{\perp}$. It is a very effective method as it gives good convergence and the results do not depend on $\Delta_{\perp \text { max }}$. 

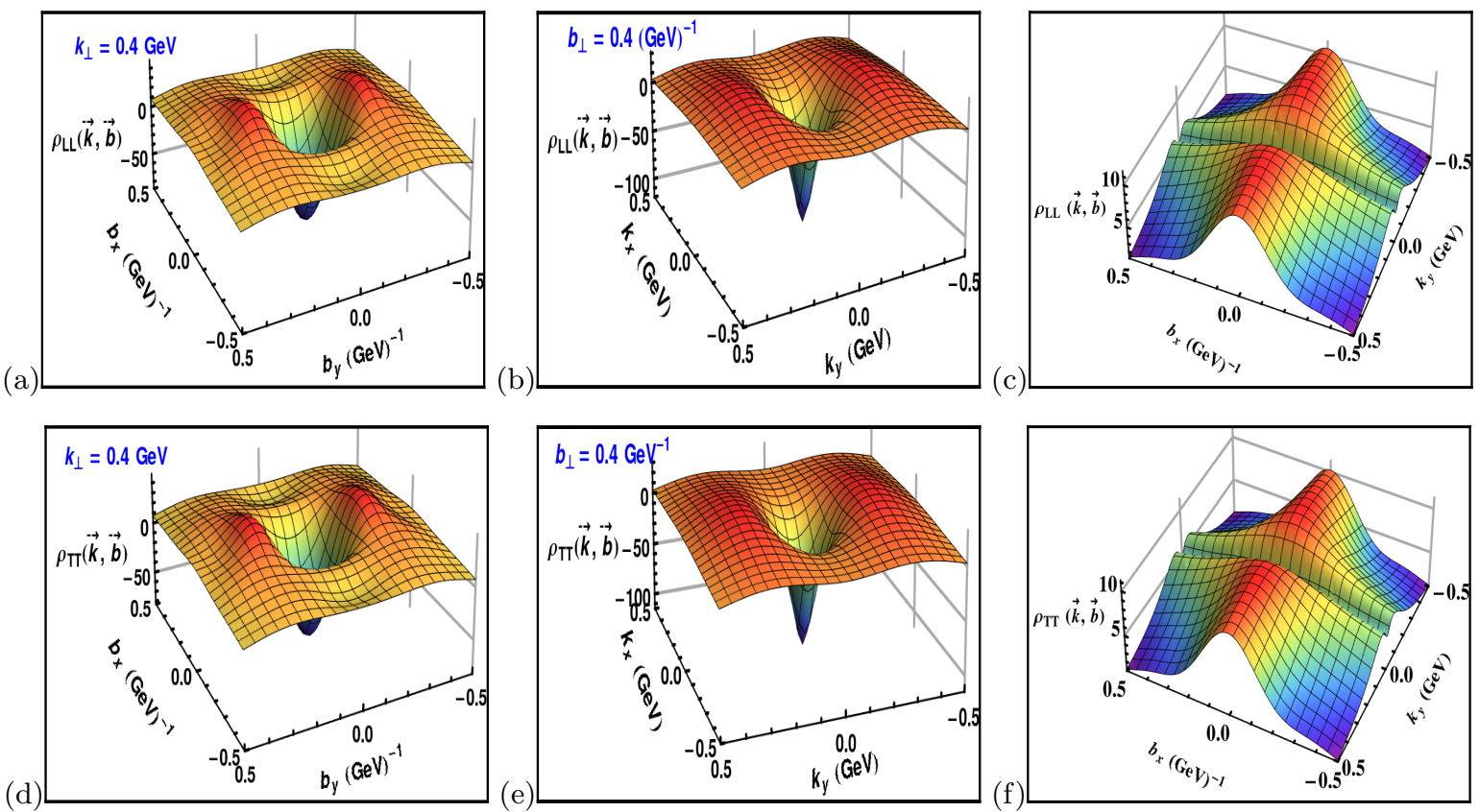

Fig. $23 \mathrm{D}$ plot of Wigner distribution $\rho_{L L}$ and $\rho_{T T}$ in $\boldsymbol{k}_{\perp}$-space, $\boldsymbol{b}_{\perp}$-space and $b_{x}-k_{y}$ space with $\Delta_{\perp \max }=$ $20 \mathrm{GeV}$

For numerical evaluation, in all the plots we have taken $m=0.33 \mathrm{GeV}$ and $\Delta_{\perp \max }=20$ GeV. Fig 1(a) show a three-dimensional plot of Wigner distribution $\rho_{U U}$ in $\boldsymbol{k}_{\perp}-$ space with $\boldsymbol{b}_{\perp}=$ $0.4 \mathrm{GeV}^{-1} \hat{\boldsymbol{e}}_{y} \cdot \rho_{U U}$ has a minimum and pick up negative value at $k_{x}=0$ and $k_{y}=0$. Fig 1 (b) show the Wigner distribution $\rho_{U U}$ in $\boldsymbol{b}_{\perp}$-space with $\boldsymbol{k}_{\perp}=0.4 \mathrm{GeV} \hat{\boldsymbol{e}}_{y}$. The behavior of $\boldsymbol{k}_{\perp}-$ space is similar to $\boldsymbol{b}_{\perp}$-space with a different minima at $b_{x}=0$ and $b_{y}=0$ but it is still negative. Fig 1 (c) show the Wigner distribution $\rho_{U U}$ in mixed space where we have integrated out $k_{x}$ and $b_{y}$ dependence, thus we get the probability densities in the $b_{x}-k_{y}$ plane.

Fig. 1(d) shows a three-dimensional plot of Wigner distributions $\rho_{U L}$ in momentum space with $\boldsymbol{b}_{\perp}=0.4 \mathrm{GeV}^{-1} \hat{\boldsymbol{e}}_{y}$. Fig. 1(e) shows a three-dimensional plot of Wigner distributions $\rho_{U L}$ in impact parameter space with $\boldsymbol{k}_{\perp}=0.4 \mathrm{GeV} \hat{\boldsymbol{e}}_{y}$. Figs. 1(d) and (e) have similar nature. These two graphs show dipole structure as observed in Ref 9; 14. Fig. 1(f) shows a three-dimensional plot of Wigner distributions in mixed space which shows the quadruple structure.

Fig 2(a)-(c) shows the three-dimensional plot of Wigner distributions $\rho_{L L}$ in $\boldsymbol{k}_{\perp}-$ space, $\boldsymbol{b}_{\perp}-$ space and the mixed space respectively. Fig. 2 for $\rho_{L L}$ shows similar nature as Fig 1 for $\rho_{U U}$ as expected.

Fig. 2(d)-(f) shows the three-dimensional plot of Wigner distribution $\rho_{T T}$ in $\boldsymbol{k}_{\perp}-$ space, $\boldsymbol{b}_{\perp}-$ space and mixed space respectively. In this case, both the quark and the target state are transversely polarized in the direction say $x$-direction. It is important to note that nature of $\rho_{T T}$ is similar to $\rho_{U U}$ and $\rho_{L L}$.

\section{Conclusion}

In this work, we include the transverse polarization of the quark and the target state unlike in the previous work [14. In this model, we study only 4 independent quark Wigner distributions as illustration and the details will be given in Ref [23]. The unpolarized $\rho_{U U}$, longitudinally polarized $\rho_{L L}$ and transversity distributions $\rho_{T T}$ show similar nature. $\rho_{L U}$ is equal to $\rho_{U L}$, which is related to orbital angular momentum of the quark and the pretzelous Wigner distribution $\rho_{T T}^{\perp}$ is zero. We have used an improved method of numerical integration that gives better convergence and the results are independent of $\Delta_{\perp \max }$.

Acknowledgements J.M. would like to thank Science and Engineering Research Board(SERB), for providing financial support and Light Cone organizers for their kind hospitality. 


\section{References}

1. S. Meissner, A. Metz and M. Schlegel, JHEP 0908, 056 (2009).

2. A. V. Radyushkin, Phys. Rev. D 56, 5524 (1997).

3. X. D. Ji, Phys. Rev. Lett. 78, 610 (1997).

4. S. Meissner, A. Metz, M. Schlegel and K. Goeke, JHEP 0808, 038 (2008).

5. C. Lorcé and B. Pasquini, JHEP 1309, 138 (2013).

6. X. D. Ji, Phys. Rev. Lett. 91, 062001 (2003).

7. A. V. Belitsky, X. D. Ji and F. Yuan, Phys. Rev. D 69, 074014 (2004).

8. E. P. Wigner, Phys. Rev. 40, 749 (1932).

9. C. Lorcé and B. Pasquini, Phys. Rev. D 84, 014015 (2011).

10. C. Lorcé, B. Pasquini, X. Xiong and F. Yuan, Phys. Rev. D 85, 114006 (2012).

11. P. Hagler, A. Mukherjee and A. Schafer, Phys. Lett. B 582, 55 (2004).

12. K. Kanazawa, C. Lorcé, A. Metz, B. Pasquini and M. Schlegel, Phys. Rev. D 90, no. 1, 014028 (2014).

13. A. Rajan, A. Courtoy, M. Engelhardt and S. Liuti, Phys. Rev. D 94, no. 3, 034041 (2016).

14. A. Mukherjee, S. Nair and V. K. Ojha, Phys. Rev. D 90, no. 1, 014024 (2014).

15. A. Mukherjee, S. Nair and V. K. Ojha, Phys. Rev. D 91, no. 5, 054018 (2015).

16. C. Lorcé, B. Pasquini and M. Vanderhaeghen, JHEP 1105, 041 (2011).

17. T. Liu, arXiv:1406.7709 [hep-ph].

18. T. Liu and B. Q. Ma, Phys. Rev. D 91, 034019 (2015).

19. G. A. Miller, Phys. Rev. D 90, no. 11, 113001 (2014).

20. C. Lorcé and B. Pasquini, Phys. Rev. D 93, no. 3, 034040 (2016).

21. A. Harindranath, R. Kundu and W. M. Zhang, Phys. Rev. D 59, 094012 (1999), Phys. Rev. D 59, 094013 (1999).

22. W. M. Zhang and A. Harindranath, Phys. Rev. D 48, 4881 (1993).

23. J. More, A. Mukherjee and S. Nair, in preparation. 